\section{Neural Network Decision Directed Edge-Adaptive Kalman Filter for Image Estimation}

\author{
Mahmood R. Azimi-Sadjadi, Rongrui Xiao, and Xi Yu
}

\begin{abstract}
A neural network-based scheme for decision directed edgeadaptive Kalman filtering is introduced in this work. A backpropagation neural network makes the decisions about the orientation of the edges based on the information in a window centered at the current pixel being processed. Then based upon the neural network output an appropriate image model which closely matches the local statistics of the image is chosen for the Kalman filter. This prevents the oversmoothing of the edges, which would have otherwise been caused by the standard Kalman filter. Simulation results are presented which show the effectiveness of the proposed scheme.
\end{abstract}

Index Terms - Image restoration, Kalman filtering, neural networks.

\section{INTRODUCTION}

Adaptive Kalman filtering schemes which use spatial-varying image models [1]-[3] take into account the local statistical information within a processing window and thus preserve edges with a greater noise reduction in nonedge regions. This obviously leads to a processed image with better visual appearance. In [1], an edge-adaptive Kalman filter was introduced that uses multiple image models to reduce the ringing artifacts caused by spatial-invariant filters. The selection of the appropriate model is done using the maximum a posteriori (MAP) method. A similar idea is exploited in this paper to develop a neural network-based decision directed edgeadaptive Kalman filter. An edge detection method for noisy images is developed using the stochastic gradient [4] algorithm. To classify the detected edges of the image into five classes corresponding to $0,45,90,135^{\circ}$ edge orientation and nonedge (background), a backpropagation neural network (BPNN) is used. The selection of the image model can then be made based upon the output of the network. A bank of five reduced update Kalman filter (RUKF) [5], [6] is used to perform the filtering operation. The combined results of these filters when used in conjunction with the BPNN provide restored images with substantially improved quality.

\section{BPNN FOR EDGE ClAsSIFICATION}

Before using the neural network to perform edge classification from a noisy image, preprocessing needs to be done in order to efficiently extract the edge information. The stochastic gradient method [4] is used here owing to its robustness in presence of noise. The gradients are calculated by using the forward and backward estimates of the image based on the noisy observations. A $3 \times 3$ window is swept pixel by pixel across the image and an edge extracted map is generated. The extracted edge map of the noisy image in each window can then be applied as the input to the neural network, which classifies them into one of the five possible classes namely nonedge (background) and four edge classes for $0,45,90$, and $135^{\circ}$ edge orientations. A three-layer BPNN [7] with 9-15-25-5

Manuscript received June 26, 1997; revised July 16, 1998. The associate editor coordinating the review of this manuscript and approving it for publication was Prof. Stephen E. Reichenbach.

The authors are with the Department of Electrical Engineering, Colorado State University, Fort Collins, CO 80523 USA (e-mail: azimi@engr.colostate.edu).

Publisher Item Identifier S 1057-7149(99)02675-5. structure was used for this classification task. The training set for this network consisted of different possible edge patterns. To guarantee the generalization of the BPNN, noisy edge patterns were also included in the training set. A total of 260 cases were selected as the training patterns for all the five edge types with 40 for each of the $0,45,90$, and $135^{\circ}$ cases, and 100 for the nonedge case. The BPNN converged to a predefined error goal (0.01), after about 22000 training epochs. The trained BPNN was then used in conjunction with the RUKF to perform edge adaptive filtering.

It is worthwhile to mention the relationship between our BPNNbased edge classification scheme and the MAP-based method in [1]. In the BPNN training, the minimum squared error (MSE) criterion is used and the desired output for an input belonging to class $k$ is set to one, while the desired outputs for the other output neurons are zero. If the global minimum is reached in the training, then it has been shown in [8] that the output of the neural network would provide the a posteriori probability of each class. Consequently, the performance of the BPNN reaches that of MAP which is optimal. However, the main benefit of using BPNN-based method over the MAP classifier is that one does not need to assume Gaussian distributions. Furthermore, BPNN offers better generalization in presence of high level noise and the computational cost is much lower after the training is completed.

\section{Reduced Update Kalman Filter (RUKF)}

The RUKF [5], [6] is a scalar minimum variance estimator which under the constraint that the updating occurs on the closest neighbors provides suboptimal estimates very efficiently.

Assume that the original image, $s(m, n)$, is modeled by a firstorder 2-D autoregressive (AR) process with a nonsymmetric half plane (NSHP) region of support [5], [6], i.e.

$$
\begin{aligned}
s(m, n)= & c_{1,0} s(m-1, n)+c_{-1,1} s(m+1, n-1) \\
& +c_{0,1} s(m, n-1)+c_{1,1} s(m-1, n-1) \\
& +w(m, n)
\end{aligned}
$$

where $c_{i, j}^{\prime}$ s are the model coefficients, and $w(m, n)$ is a zero-mean white Gaussian random field with variance $\sigma_{w}^{2}$ which drives the process. The scalar observation model for the no blur case is

$$
r(m, n)=s(m, n)+v(m, n)
$$

where $v(m, n)$ represents the observation noise which is also a white Gaussian noise, with zero-mean and variance $\sigma_{v}^{2}$. The state dynamical model and the observation equation then become [5]

$$
\begin{aligned}
& \boldsymbol{s}(m, n)=\boldsymbol{C s}(m-1, n)+\boldsymbol{w}(m, n) \\
& r(m, n)=\boldsymbol{h} \boldsymbol{s}(m, n)+v(m, n)
\end{aligned}
$$

where the "global state vector," $\boldsymbol{s}(m, n)$, consists of two parts: $\boldsymbol{s}_{\mathbf{1}}(m, n)$ which is called the "local state vector," and $\boldsymbol{s}_{\mathbf{2}}(m, n)$ which contains the remaining part of $\boldsymbol{s}(m, n)$. Fig. 1 shows the regions of support for $\boldsymbol{s}_{\mathbf{1}}(m, n)$ and $\boldsymbol{s}_{\mathbf{2}}(m, n)$ in the image for a first-order two-dimensional (2-D) NSHP model. Thus, we have

$$
\boldsymbol{s}(m, n)=\left[\boldsymbol{s}_{\mathbf{1}}^{t}(m, n), \boldsymbol{s}_{\mathbf{2}}^{t}(m, n)\right]^{t}
$$




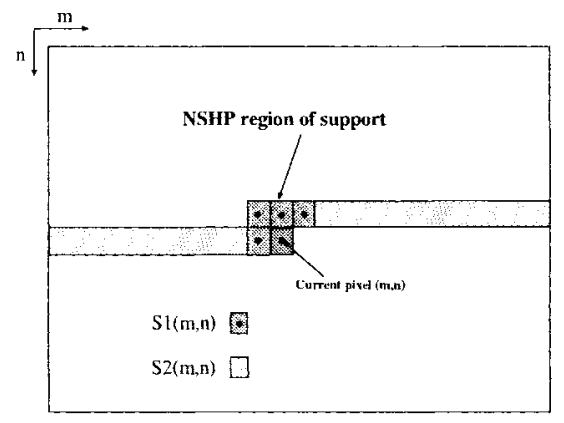

Fig. 1. Local and global states and region of support for the image model.

where $\boldsymbol{s}_{\mathbf{1}}(m, n)$ and $\boldsymbol{s}_{\mathbf{2}}(m, n)$ are defined as

$$
\begin{aligned}
\boldsymbol{s}_{\mathbf{1}}(m, n)= & {[s(m, n), s(m-1, n), s(m+1, n-1),} \\
& s(m, n-1), s(m-1, n-1)]^{t} \\
\boldsymbol{s}_{\mathbf{2}}(m, n)= & {[s(m-2, n), s(m-3, n), \cdots, s(1, n) ; .} \\
& s(N, n-1), s(N-1, n-1), \\
& \cdots, s(m+2, n-1)]^{t}
\end{aligned}
$$

Also

$$
\begin{aligned}
\boldsymbol{w}(m, n) & =[w(m, n), 0,0,0, \cdots, 0]^{t} \\
\boldsymbol{h} & =[1,0,0,0, \cdots, 0] \\
\boldsymbol{C} & =\left[\begin{array}{ll}
C_{11} & C_{12} \\
C_{21} & \boldsymbol{C}_{22}
\end{array}\right]
\end{aligned}
$$

The constituent blocks in matrix $C$, i.e., $C_{11}, C_{12}$ contain all $c_{i}$ terms, and $\boldsymbol{C}_{21}, \boldsymbol{C}_{22}$ contain only zeros and ones for shift operations. They are defined as follows:

$$
\begin{aligned}
\boldsymbol{C}_{11} & =\left[\begin{array}{ccccc}
c_{1,0} & 0 & c_{0,1} & c_{1,1} & 0 \\
1 & 0 & 0 & 0 & 0 \\
0 & 0 & 0 & 0 & 0 \\
0 & 0 & 1 & 0 & 0 \\
0 & 0 & 0 & 1 & 0
\end{array}\right] ; \\
\boldsymbol{C}_{12} & =\left[\begin{array}{cccc}
0 & 0 & \cdots & c_{-1,1} \\
0 & 0 & \cdots & 0 \\
0 & 0 & \cdots & 1 \\
0 & 0 & \cdots & 0 \\
0 & 0 & \cdots & 0
\end{array}\right] \\
\boldsymbol{C}_{21} & =\left[\begin{array}{ccccc}
0 & 1 & 0 & 0 & 0 \\
0 & 0 & 0 & 0 & 0 \\
0 & 0 & 0 & 0 & 0 \\
\vdots & \vdots & \vdots & \vdots & \vdots \\
0 & 0 & 0 & 0 & 0
\end{array}\right] ; \quad \boldsymbol{C}_{22}=\left[\begin{array}{cccc}
0 & 0 & \cdots & 0 \\
1 & 0 & \cdots & 0 \\
0 & 1 & \cdots & 0 \\
\vdots & \vdots & \ddots & \vdots \\
0 & 0 & \cdots & 1
\end{array}\right] .
\end{aligned}
$$

The Kalman gain vector and the a priori and a posteriori error covariance matrices are also partitioned in a similar manner [1]. Note that the Kalman gain vector has only five nonzero elements in this case. The RUKF [5], [6] for the scalar observation and in presence of additive noise alone can then be applied to estimate the image. This filtering procedure can easily be extended to the general image restoration problem by modifying the observation equation to incorporate the point spread function (PSF) of the blur [1], [6].

\section{Edge-Adaptive Kalman Filtering Scheme}

The block diagram of the BPNN-based decision directed edgeadaptive Kalman filtering scheme is depicted in Fig. 2 which shows a bank of RUKF's that activate by the BPNN edge classifier. For each edge scenario, a first-order 2-D AR model with different set

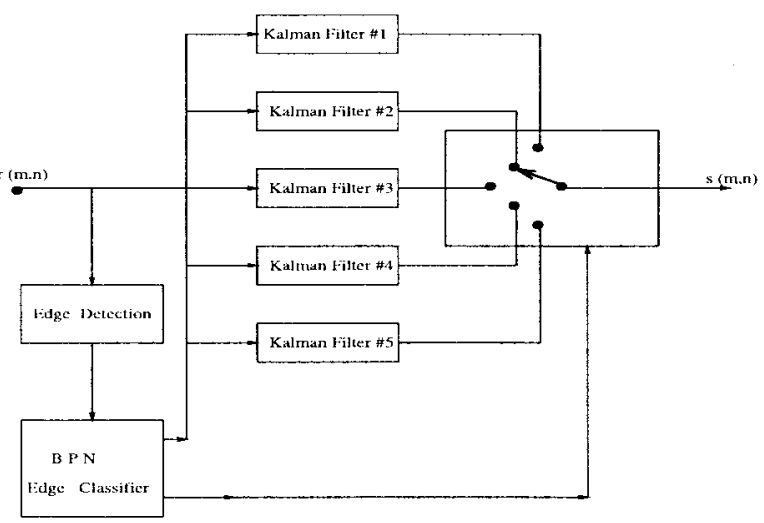

Fig. 2. BPNN decision directed edge-adaptive Kalman filtering scheme.

TABLE I

AR Model Coefficients for Five Edge Orientations [5]

\begin{tabular}{c|c|c|c|c|c|c}
\hline \hline$I_{\text {edge }}$ & Edge & \multicolumn{5}{|c}{ Model Coefficients } \\
\cline { 3 - 7 } & Orientation & $c_{1}$ & $c_{2}$ & $c_{3}$ & $c_{4}$ & $\sigma_{1}^{2}$ \\
\hline 1 & $0^{\circ}$ & 0.96977 & 0.00643 & -0.00180 & 0.02461. & 32.2181 \\
\hline 2 & $15^{\circ}$ & 0.01819 & 0.96874 & $0.0 ! 183$ & 0.00088 & 32.1013 \\
\hline 3 & $90^{\circ}$ & -0.00263 & 0.03650 & 0.925 .56 & 0.03989 & 31.8146 \\
\hline 1 & $135^{\circ}$ & 0.03029 & 0.00085 & 0.02923 & 0.93956 & 31.2378 \\
\hline 5 & non-edge & 0.24573 & 0.24430 & 0.246 .58 & 0.26247 & 32.20335 \\
\hline
\end{tabular}

of coefficients is chosen. In [1], five sets of AR model coefficients were computed for a test image. These coefficients are used here to generate an edge-dependent model, i.e.,

$$
\begin{aligned}
s(m, n)= & c_{1,0}\left(I_{\text {edge }}\right) s(m-1, n) \\
& +c_{-1,1}\left(I_{\text {edge }}\right) s(m+1, n-1) \\
& +c_{0,1}\left(I_{\text {edge }}\right) s(m, n-1) \\
& +c_{1,1}\left(I_{\text {edge }}\right) s(m-1, n-1)+w(m, n)
\end{aligned}
$$

for the adaptive Kalman filtering, where $I_{\text {edge }}$ is an integer number between one and five, corresponding to the five different edge types mentioned above. Table I gives $c_{i, j}\left(I_{\text {edge }}\right)$ values and variance of the white noise $w(m, n)$ for each case.

After normalization, the content of the window will be applied to the BPNN to perform the classification. According to the value of $I_{\text {edge }}$ determined based upon the edge presence and orientation, the corresponding image model for the RUKF will be chosen to perform the filtering operation. The procedure is repeated for all the pixels in the image.

Both the BPNN and MAP-based edge-adaptive Kalman filters were applied to the Lena images in Fig. 3 which is corrupted by additive noise to generate $\mathrm{SNR}=5.2 \mathrm{~dB}$. For the MAP-based edge classification scheme, as suggested in [1], the priori probabilities for different edge scenario are computed by finding their relative frequency over the entire image. The resulting probabilities were found to be $0.03,0.02,0.12,0.15$, and 0.68 corresponding to 0 , $45,90,135^{\circ}$ and nonedge region, respectively for this test image. Fig. 4 shows the processed image using the MAP-based edge adaptive Kalman filter. The SNR for this image was measured to be $8.7 \mathrm{~dB}$. The processed image of the BPNN-based edge adaptive Kalman filter is shown in Fig. 5. The SNR for this image was found to be 10.7 $\mathrm{dB}$ which indicates $3 \mathrm{~dB}$ improvement over the result in Fig. 4. The visual comparison of the two images also reveal the fact that the proposed BPNN-based edge-adaptive Kalman filtering scheme removes the noise more efficiently without causing considerable 


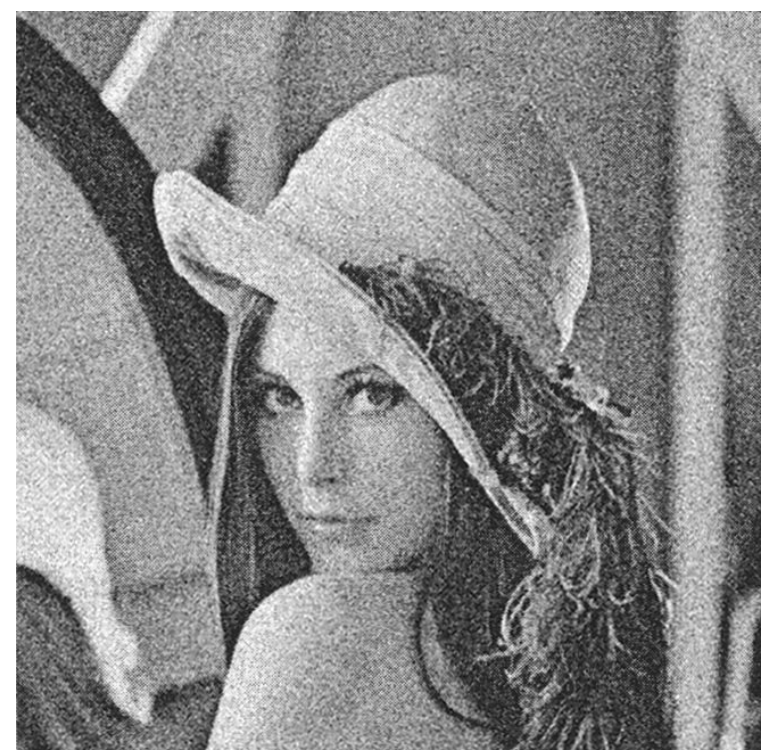

Fig. 3. Noisy Lena image, $\mathrm{SNR}=5.2 \mathrm{~dB}$.

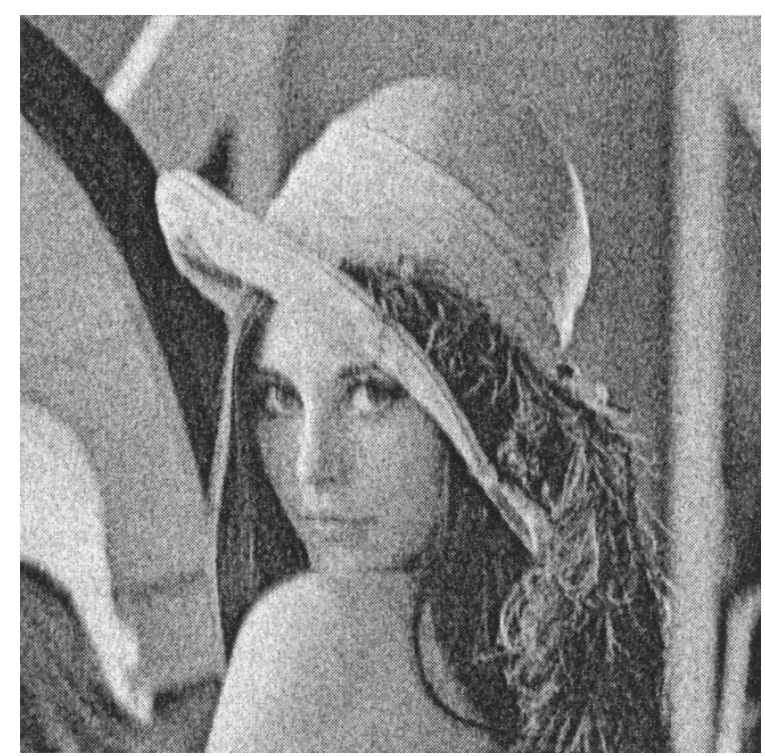

Fig. 4. MAP-based edge adaptive RUKF processed image, $\mathrm{SNR}=8.7 \mathrm{~dB}$.

blurring artifacts. A standard RUKF with the same region of support was also applied to the noisy test image. In this case, the AR model coefficients were obtained using the Yule-Walker method [4]. The coefficients of this model were estimated to be $c_{1,0}=$ $0.5453, c_{-1,1}=0.1003, c_{0,1}=0.6390, c_{1,1}=-0.2823$, and the variance of the driving process was $\sigma_{w}^{2}=41.9962$. The processed image for this case is shown in Fig. 6. As can be seen, the BPNNbased edge adaptive RUKF provides $2.5 \mathrm{~dB}$ SNR improvement over the standard RUKF filter.

Fig. 7 shows the performance comparison of the BPNN and MAPbased edge adaptive Kalman filtering schemes for different SNR values of the original image namely 1, 5.2, 10, 20, 30, and $40 \mathrm{~dB}$. The upper curve corresponds to the BPNN-based method. As can be observed from these operating characteristic plots, the BPNN-based adaptive Kalman filter provides better SNR improvement, especially at lower SNR. This is due to the fact that the training set used to train the BPNN consisted of several noisy edge patterns. Consequently, this

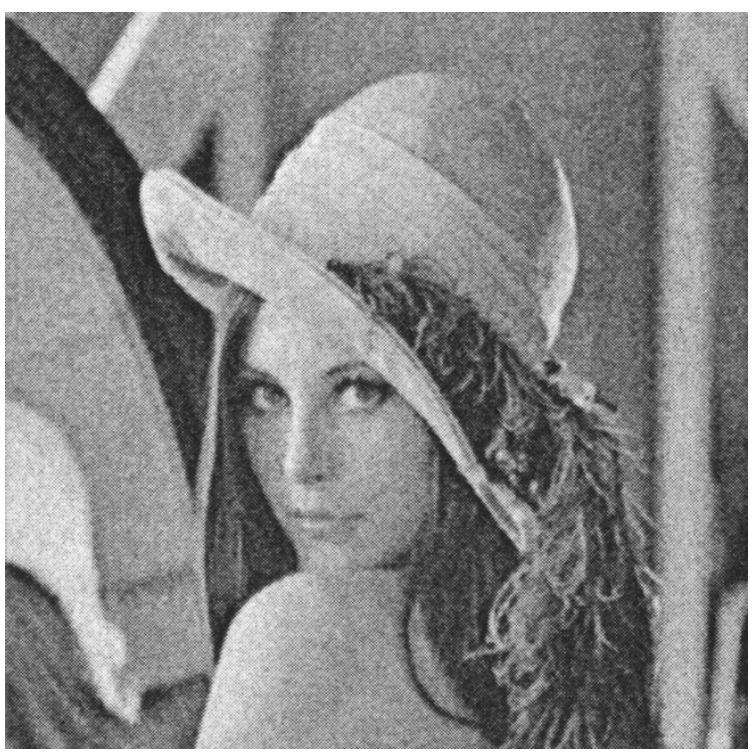

Fig. 5. BPNN-based edge adaptive RUKF processed image, SNR $=10.7 \mathrm{~dB}$.

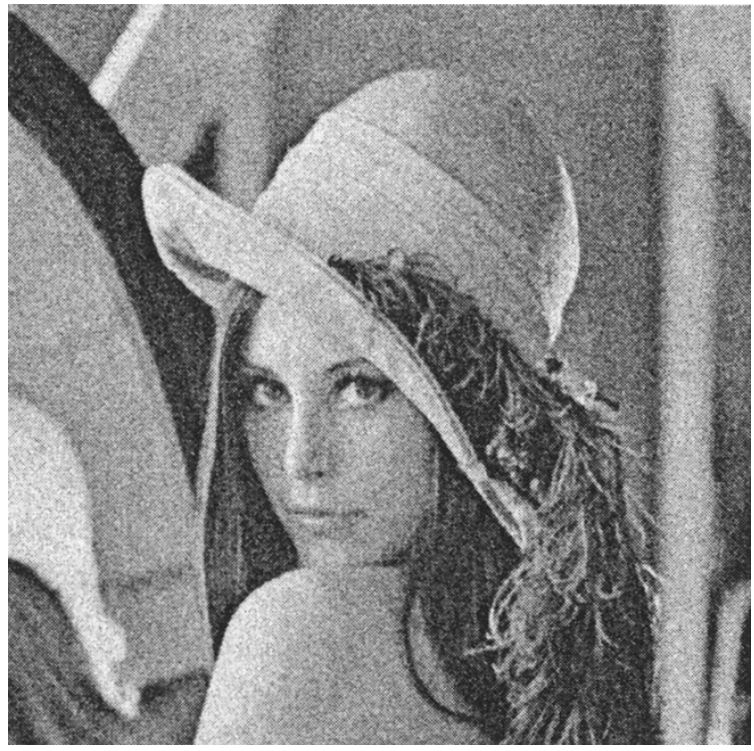

Fig. 6. Standard RUKF processed image, $\mathrm{SNR}=8.2 \mathrm{~dB}$.

leads to better generalization and thus better noise tolerance of this scheme over the MAP-based method.

Finally, to study the relationship between the number of classes, and SNR performance improvement several experiments were conducted. Table II gives the results of the BPNN-based edge adaptive Kalman filter using different edge dependent RUKF's. The first five cases in this table correspond to a single class case where only one edge dependent RUKF is used to process the image. In these cases, the highest SNR was obtained for a nonedge case. The reason being the nonedge regions form most of the image, with edge blocks making up only a small part of the whole image. For the sixth case in the table, three edge classes namely $0,90^{\circ}$ and nonedge were allowed leading to a bank of three RUKF's. The SNR of the resultant image for this case was only $0.22 \mathrm{~dB}$ better than the nonedge case. This slight improvement is due to the addition of the two edge directions, i.e., $0,90^{\circ}$. When the number of classes increased from three to five, an additional $0.05 \mathrm{~dB}$ improvement was achieved as evident from the 


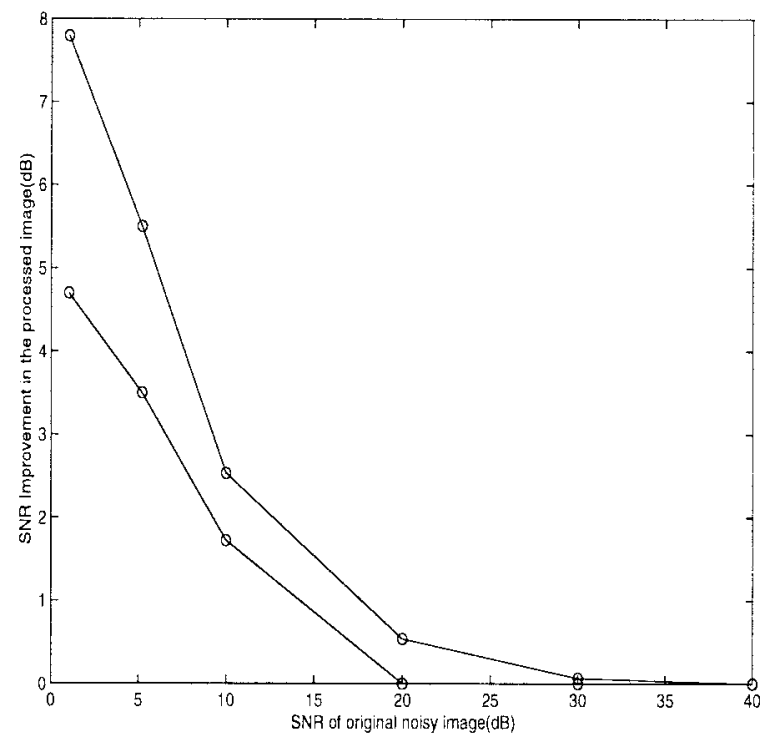

Fig. 7. Performance comparison of the BPNN and MAP-based edge adaptive Kalman filters.

TABLE II

Relationship Between the Number of Classes And the SNR Performance

\begin{tabular}{|c|c|c|c|c|c|}
\hline \multicolumn{5}{|c|}{ Edge dependent RUKF } & \multirow{2}{*}{$\begin{array}{l}\text { Performance } \\
\text { (SNR) }\end{array}$} \\
\hline$\overline{0^{\circ}}$ & $45^{\circ}$ & $90^{\circ}$ & $135^{\circ}$ & non-edge & \\
\hline $\mathrm{x}$ & & & & & 6.5231 \\
\hline & $\mathrm{X}$ & & & & 7.6004 \\
\hline & & $\mathrm{X}$ & & & 7.7800 \\
\hline & & & $\overline{\mathrm{X}}$ & & 7.6331 \\
\hline & & & & $\mathrm{X}$ & $10.45 \overline{02}$ \\
\hline $\mathrm{x}$ & & $\mathrm{X}$ & & $\mathrm{X}$ & 10.6684 \\
\hline $\mathrm{X}$ & $\mathrm{X}$ & $\bar{X}$ & $\mathrm{x}$ & $X$ & 10.7166 \\
\hline
\end{tabular}

result in the last row of Table II. This improvement is much lower than the case from one class to three classes. As a result, one can not expect to get noticeable improvement by increasing the number of the classes beyond five. In addition, increasing the number of class increases the computational time. Thus, five classes appear to be the optimal choice for this problem.

\section{CONCLUSION}

A neural network-based decision directed edge-adaptive Kalman filtering scheme is introduced in this paper. A BPNN makes decisions based on the noisy edge information in a window centered at the current pixel being processed. Once the edge orientation is determined, an image model for Kalman filter can be chosen to closely match the local statistics of the image. Simulation results are provided which show the effectiveness of our scheme for noise removal without causing noticeable smearing of the edges. A detail comparison with the MAP-based edge adaptive Kalman filter and the standard Kalman filter was also provided.

\section{REFERENCES}

[1] A. M. Tekalp, H. Kaufman, and J. W. Woods, "Edge-adaptive Kalman filtering for image restoration with ringing suppression," IEEE Trans. Acoust., Speech, Signal Processing, vol. 37, pp. 892-899, June 1989.
[2] M. R. Azimi-Sadjadi and S. Bannour, "Two-dimensional recursive parameter identification for adaptive Kalman filtering," IEEE Trans. Circuits Syst., vol. 38, pp. 1077-1081, Sept. 1991.

[3] R. Wilson, H. E. Knutsson, and G. H. Granlund, "Anisotropic nonstationary image estimation and its application: Part II Predictive image coding," IEEE Trans. Commun., vol. 31, pp. 398-406, Mar. 1983.

[4] A. K. Jain, Fundamentals of Digital Image Processing. Englewood Cliffs, NJ: Prentice-Hall, 1989.

[5] J. W. Woods and C. H. Radewan, "Kalman filtering in two dimensions," IEEE Trans. Inform. Theory, vol. IT-23, pp. 473-482, July 1977.

[6] J. W. Woods and V. K. Ingle, "Kalman filtering in two dimensions: Further results," IEEE Trans. Acoust., Speech, Signal Processing, vol. ASSP-29, pp. 188-197, Apr. 1981.

[7] J. A. Freeman and D. M. Skapura, Neural Networks: Algorithms, Applications, and Programming Techniques. Reading, MA: AddisonWesley, 1991.

[8] N. Hermann, "On the probabilistic interpretation of neural network classifiers and discriminative training criteria," IEEE Trans. Pattern Anal. Machine Intell., vol. 17, pp. 107-119, Feb. 1995.

\section{Statistical Texture Characterization from Discrete Wavelet Representations}

G. Van de Wouwer, P. Scheunders, and D. Van Dyck

Abstract-We conjecture that texture can be characterized by the statistics of the wavelet detail coefficients and therefore introduce two feature sets: 1) the wavelet histogram signatures which capture all first order statistics using a model based approach and 2) the wavelet co-occurrence signatures, which reflect the coefficients' second-order statistics.

The introduced feature sets outperform the traditionally used energy. Best performance is achieved by combining histogram and co-occurrence signatures.

Index Terms - Feature extraction, multiscale representation, texture analysis, wavelets.

\section{INTRODUCTION}

Texture analysis plays an important role in many image processing tasks, ranging from remote sensing to medical imaging, robot vision and query by content in large image databases. Various methods for texture feature extraction have been proposed during the last decades (e.g., [1]), but the texture analysis problem remains difficult and subject to intensive research.

A major class of feature extractors relies on the assumption that texture can be defined by the local statistical properties of pixel gray levels. From the image histogram, first-order statistics can be derived and used as texture features. It was soon argued that they did not suffice for adequate texture description and that second-order statistics were required, as efficiently reflected in features computed from the co-occurrence matrix [2]. The conjecture that second-order statistics suffice for texture analysis was later rejected [3] and various other texture analysis schemes were introduced (e.g., based on Markov

Manuscript received June 30, 1997; revised June 25, 1998. The associate editor coordinating the review of this manuscript and approving it for publication was Prof. Kannan Ramchandran.

The authors are with the Vision Laboratory, Department of Physics, University of Antwerp, 2020 Antwerpen, Belgium (e-mail: wouwer@ruca.ua.ac.be).

Publisher Item Identifier S 1057-7149(99)02451-3. 